\title{
Changes in Measures of Dementia Awareness in UK Church Congregations Following a 'Dementia- Friendly' Intervention: A Pre-Post Cohort Study
}

\author{
Peter Kevern ${ }^{1, *(1)}$ and David Primrose ${ }^{2}$ \\ 1 Professor of Values in Health and Social Care, Staffordshire University, Stoke-on-Trent, ST4 2DE, UK \\ 2 Transforming Church and Communities Enabler, Anglican Diocese of Lichfield, Lichfield, WS13 7LD, UK; \\ david.primrose@lichfield.anglican.org \\ * Correspondence: p.kevern@staffs.ac.uk
}

Received: 2 June 2020; Accepted: 29 June 2020; Published: 7 July 2020

\begin{abstract}
Given their stability, their ability to influence public attitudes and capacity to mobilise human resources, it seems likely that many faith communities can have a significant impact on the wellbeing of people living with dementia (PLWD) and their carers, who may be dependent on the commitment of informal communities of support over months or years. This paper reports on a pre-post cohort study undertaken in an Anglican (Church of England) diocese in the UK. Representatives $(\mathrm{N}=61)$ of 11 church communities completed the Dementia Awareness Scale immediately before and 12 months after an intervention to promote 'Dementia-Friendly Church' in their congregation. Results showed a robust improvement in scores on both the 'Knowledge' and 'Comfort' subscales, with the largest effect size in the latter. Within each subscale, improvements were evenly distributed across the individual items. These findings indicate that such an intervention is likely to significantly improve the environment for people living with dementia and their companions who are members of or engage with the Church community. They also suggest that a similar programme could be extended to other intentional communities, whether in faith-based organisations or societies with shared interests.
\end{abstract}

Keywords: Dementia-Friendly Church; Alzheimer's; religion; quantitative; cohort study

\section{Introduction}

'Dementia-Friendly Communities' are now an established feature of the collective response to dementia, with 349 registered in England alone at the time of writing (Alzheimer's Society 2020) and a global movement to establish them promoted by Alzheimer Disease International (Alzheimer Disease International 2016). The purpose of the movement is to bring about a cultural change at the community level that will change the experience of living in the community for people living with dementia (PLWD) and their carers. Typically, intervention will centre on eliciting the support of local businesses for changes that will reduce the stress and increase the support for PLWD, along with an exercise in whole-community education about dementia and support for PLWD.

Of the current 349 recognised Dementia-Friendly Communities, almost all are geographically or territorially defined, ranging in size from metropolitan cities to small hamlets. Only four stand out, as defining intentional or occupational communities which may cross geographical boundaries with their network of relationships: the University of Nottingham and three groups of Christian churches. This represents a missed opportunity: churches may have particular potential in the support of PLWD and their carers because they have a permanence that many interventions lack. For example, PLWD and their carers frequently report problems with continuity of support: specialists may know only 
part of the story and frequently change; there may be a disconnect between health care and social services; support may be offered for a limited period and then expire. At a more fundamental level, stigma may limit social interaction (Department of Health 2015). By contrast, faith-based communities tend to be stable over long periods of time and committed in the long term to those who join or visit them, they tend to work with family networks as well as individuals, to be able to mobilise voluntary support and to either challenge or reinforce particular attitudes which impede the establishment of a society valuing people living with dementia (Post 2000; Kevern 2009; Swinton 2017). In Goldenberg's evocative terminology, they function as 'vestigial states', parallel or alternative social orders that can supply some of the shortcomings of the secular social order (Goldenberg 2015); and, in theory at least, these features make them particularly useful in supporting PLWD and their carers (Márquez-González et al. 2012; Farr and Bute 2015; Jewell et al. 2016, 2017).

The idea of 'Dementia-Friendly Church' (DFCh) has emerged as an extension of the Dementia-Friendly Communities movement. Its premise is that churches could be made more hospitable and welcoming places for people living with dementia and their carers by, in the first instance, a consciousness-raising exercise. By raising the knowledge and awareness of the church members and making small practical changes to the layout, worship and hospitality available in the church building (such as by improving the information available to them and by improving the physical design), the intention is to improve the experience of people living with dementia and their carers when accessing the resources of the church, and eventually by focused, practical action to change the culture of the local community and the way it is experienced by PLWD and their carers.

However, despite the popularity and profile of the Dementia-Friendly Communities model in the UK and elsewhere, there is little evidence to support its effectiveness (Heward et al. 2017). What research exists is almost all qualitative and exploratory (Dean et al. 2015a, 2015b; Institute of Public Care 2015; Lin 2017; Hebert and Scales 2019) and lacks the generalisability that may be achievable using quantitative methods. Furthermore, where evaluation had taken place, there was "little evidence of it having effected change" (Buckner et al. 2019, p. 1235). But when it comes to considering the potential for Dementia-Friendly Churches, while there is growing support for the concept (Plunkett and Chen 2016; Garland 2017), the data are even more scarce than for Dementia-Friendly Communities as a whole. A protocol for a study of Black African Churches in the USA has been published (Epps et al. 2019) and, more recently, some initial qualitative data on what 'stakeholders' (but not people living with dementia) thought a Dementia-Friendly Church may look like (Epps et al. 2020). But apart from the difficulty of translating findings from the very different religious and ethnic environments of the USA, there is little here that casts light on the particular needs of people living with dementia, or how churches may address them.

The current research, therefore, addresses a deficit in the knowledge base on Dementia-Friendly Churches by arriving at some generalisable findings and thus provides some robust recommendations for future practice. It will analyse the potential of a Dementia-Friendly Church (DFCh) initiative to influence congregational attitudes to dementia and, by extension, the capacity of church congregations to influence the experience of people living with dementia and their companions when they engage with them. There is a wider question of the ways and degrees to which changes in a local congregation may influence the attitudes of and resources available in the geographical community of which they are a part: this, however, falls outside the scope of the present study.

\section{Background}

Religious organisations have historically provided a fundamental organising structure for human communities on all scales from the local to the global. Although this organising capacity has had mixed outcomes historically, its influence is undeniable. This makes them potentially well suited as vehicles for encouraging beneficial changes at an individual and social level. In England, the religious structure with the widest reach remains the Church of England, which in theory covers the whole of the nation with a system of parishes based upon places of worship and licensed clergy. DFCh uses this network 
to attempt to bring about changes in the lives of people living with dementia and their companions. The purpose of the present study is to gather preliminary evidence regarding the degree to which this aim is realised using the current model of DFCh at a diocesan level, with a view to considering its potential for wider dissemination in faith-based organisations and other 'intentional' communities.

The diocese in which this study is based covers some 1750 square miles and a population of about 2 million. The church community numbers about 44,000 , based around 424 parishes, served by over 500 clergy in active ministry. Many church buildings serve as the centre for the local community, particularly in rural areas. Over the last seven years, in collaboration with a local university and dementia charities, the diocese has developed a process of fostering DFCh that has had wide influence in the UK and abroad. Nine years ago, discussions with local clergy identified dementia as an area of concern across the diocese, for its effects both on the congregation and on the wider community. The work of the diocese has since been recognized by the Alzheimer's Society, which in 2019 awarded it Dementia-Friendly Community status (Alzheimer's Society 2019). This in turn allows it to award Dementia-Friendly Church certificates to participating churches.

The current version of the DFCh programme uses a three-stage approach of embarking, embedding and extending.

- Embarking - In response to interest from the local church, the Dementia-Friendly Church enablers introduce material in two different ways. First, the national Dementia Friends training is delivered within a regular Sunday service. This raises awareness for the whole congregation, helps turn understanding into action and opens up conversations around the topic of dementia. Second, the DFCh training course, usually run over four sessions, is delivered to a core group of volunteers. Increasingly the training is provided simultaneously to volunteers from several churches.

- Embedding-The next stage is when the church commits to Dementia-Friendly Church status, which the diocese is able to grant through the status it has achieved as a Dementia-Friendly Community. For this, the church identifies three actions which they will undertake in the coming year. Churches are awarded Dementia-Friendly Church certificates, which are renewable each year.

- Extending-The third stage involves engaging with the wider community, partnering with others to ensure that dementia-friendly churches are at the heart of dementia-friendly communities. This can entail building strong links through Dementia Action Alliances.

As well as personal support, those running the programme provide peer support groups for dementia coordinators, produce a regular newsletter and arrange an annual conference. By the end of 2019, the programme had included 101 Dementia Friends Sunday services, making 4151 Dementia Friends. The four-session course, or equivalent, had been delivered 31 times, with 575 participants. At that time there were 70 churches with Dementia-Friendly Church certificates, setting out three actions on dementia for the coming year.

Intended outcomes of the DFCh programme are therefore on three levels:

- At the level of the local congregation, to change attitudes, improve knowledge and so enhance support for about 240 church members currently living with dementia, their family and carers. The aim is to improve both the quality of support (resources per person) and its reach (number of people supported), taking an expansive definition of 'congregation' to include the wider community and its institutions such as care homes.

- At the level of the local community, catalysing changes towards the emergence of dementia-friendly communities in collaboration with local partners and the Dementia Action Alliance.

- At the level of values, attitudes and beliefs, generating new insights into the theological and socio-political elements of dementia as experienced by members of contemporary Western society, and challenging its deep-seated values. 


\section{Research Design}

The aim of the research presented in this paper was to determine whether a church's participation in the first stage of the DFCh programme was associated with a measurable change in the responses to dementia of church attendees over a 12-month period between April 2017 and March 2019. The underlying assumption is that such changes, scaled up to the level of the congregation, amount to a change in the culture and environment encountered by people with dementia and those who share their lives when they enter the church or engage with the church community. The research adopted a repeated-measures prospective cohort design because the variability between different congregations and their contexts rendered it impractical to attempt to set up a control group.

Measurement was by means of a paper questionnaire (Figure 1), distributed with a consent form and information sheet at the initial launch event in each of the participating churches. In most cases, this was the Sunday service at which DFCh was introduced; in a few cases, it was a workshop or training event on the subject. Returned questionnaires with consent forms attached were collated at the church or mailed directly to the Principal Researcher who assigned a code to each one and detached the consent form before coding data.

1. It is rewarding to spend time with people who have dementia

2. I am afraid of people with dementia*

3. People with dementia can be creative

4. I feel confident around people with dementia

5. I am comfortable touching people with dementia

6. I feel uncomfortable being around people with dementia*

7. Every person with dementia has different needs

8. I am not very familiar with dementia *

9. I would avoid an agitated person with dementia*

10. People with dementia like having familiar things nearby

11. It is important to know the past history of people with dementia

12. It is possible to enjoy the company of people with dementia

13. I feel relaxed around people with dementia

14. 1People with dementia can enjoy life

15. People with dementia can feel when others are kind to them

16. I feel frustrated because I do not know how to help people with dementia*

17. I cannot imagine caring for someone with dementia*

18. I admire the coping skills of people with dementia

19. We can do a lot now to improve the lives of people with dementia

20. If somebody who has dementia is being 'difficult', it may be that they are trying to communicate

21. Which of these diseases do you worry about getting when you get older? [diabetes, stroke, heart disease, dementia, cancer, depression, influenza]. Put them in order from 1 (I am most worried about this) to 7 (I am not very worried about this):*

22. In this locality how many people do you know or have heard of who are living with dementia?

23. In this locality, how many people do you know or have heard of who are caring for someone who is living with dementia?

24. Roughly how many times this week have you had a conversation where the subject of dementia has come up?

25. Roughly how many times this week have you had a conversation with somebody with dementia (or suspected dementia)?

26. Roughly how many times this week have you had a conversation with somebody about their friend or relative with dementia?

Items marked * are reverse-scored.

Knowledge Subscale comprises items 1-9, 13,16,17

Comfort Subscale comprises items 10-12, 14, 15, 18, 19, 20

Fear: item 21

Figure 1. The Dementia-Friendly Churches Questionnaire. 
After 12 months, those who had participated in the first round of the study were sent a second copy of the questionnaire (with identifying code) and invited to repeat the process. These were either collected by representatives at the church itself or mailed directly in a prepaid envelope to the Principal Researcher. The research design was scrutinised and approved by the Staffordshire University Research Ethics Committee.

The main body of the questionnaire comprised the Dementia Attitudes Scale (DAS), a 20-item scale in which each item is scored on a 7-point Likert scale. The scale demonstrates acceptable reliability (total-scale Cronbach's alphas 0.83-0.85) and convergent validity with similar scales ( $\mathrm{O}^{\prime}$ Connor and McFadden 2010).

The DAS is divisible into two subscales for Knowledge and Comfort, which are interpreted through a distinctively personalist lens. Thus, the 8-item Knowledge subscale focuses on Personhood-based rather than Biomedical Knowledge of dementia, that is, an understanding of how a person with dementia may express their personhood, and what limits it. Similarly, the 12-item Comfort subscale refers specifically to Social Comfort, to the degree to which the participant feels at ease in the presence of a person living with dementia (Ebert et al. 2019). This distinctive emphasis of the DAS renders it particularly suitable as a potential measure of the willingness of a church community to accommodate people living with dementia and how this changes over the course of a year of DFCh interventions and activities. Each item was given a point score from 1 to 7 , with scale scores as the mean of the individual item scores.

In addition to the DAS, the questionnaire incorporated a 'fear of dementia' item derived from a European study investigating how concerned a sample was at the prospect of receiving a diagnosis of dementia (Blendon et al. 2012; Hattink et al. 2015). This item asked participants to rank their fear of dementia as one element in a list of seven diseases (diabetes, stroke, heart disease, dementia, cancer, depression, influenza) that they might be afraid of getting in the future. Fear of dementia was then given a score on a scale of 1 to 7 based upon where it was ranked in the list.

Finally, five questions were included which were designed to provide an estimate of the extent to which the respondent was already engaged with or exposed to people living with dementia. The intention was to explore potential correlations between respondents' (self-reported) engagement and their attitudes. However, we discovered that respondents were vague in their answers (whether because of the free-text format or because of the content of the questions themselves) and we concluded that these questions were not capable of yielding reliable data. No analysis was attempted on them, although they are included here for the sake of completeness.

The power analysis was based on an estimated medium effect size for each of the subscales. The only study known to have used this scale in a pre-post design (George et al. 2013) did not measure effect size directly, but found an average improvement in the DAS score of 16\% (9.94\% in the knowledge domain and $24.15 \%$ in the comfort domain) in their small sample, which suggests that a medium effect size (Cohen $r=0.3-0.5)$ is a plausible starting assumption for the group receiving the intervention directly. Using paired $t$-tests (one-tailed) with $\alpha=0.05$ and $\beta-1=0.80$ gives a sample size of 27 (https://www.ai-therapy.com/psychology-statistics/sample-size-calculator).

Hypothesis 1 (H1). Key hypotheses to be tested were:
(a) There is a significant change in:
(b) Knowledge,
(c) Comfort and

Hypothesis 2 (H2). Fear of Dementia as measured by the pre-post questionnaire results.

There is a correlation between the Fear of Dementia score and the reported measures of Knowledge and Comfort

In addition to these hypotheses, exploratory analysis was conducted on the salience of particular items in the DAS questionnaire. 


\section{Analysis and Results}

\subsection{Sample Characteristics}

The first round of data collection (T1) yielded a sample (N) of 271 from 17 centres. However, attrition was high and only 61 of these, representing 11 of the original 17 centres and $22.5 \%$ of the original sample, returned a second questionnaire a year later (T2). The main reasons for the high attrition are likely to be twofold. In the first place, the departure of one of the original enablers effectively prevented some of the original centres representing 80 (30\%) of the original respondents from returning data in the second round. Secondly, the second round of data collection was not linked to a collective event, and the majority of respondents were required to mail their responses directly to the researchers. This inevitably meant that there was much less encouragement to participate, resulting in a smaller return.

In order to test the effect of attrition on the sample characteristics, the mean values for individual items on the Knowledge and Comfort subscales were compared for the larger and smaller sample groups at T1 (Table 1):

Table 1. Comparison of subscale mean values for two participant groups at T1.

\begin{tabular}{ccc}
\hline & $\mathbf{N}=\mathbf{2 7 1}$ & $\mathbf{N}=\mathbf{6 1}$ \\
\hline Mean (SD, 95\% confidence interval) Knowledge Score & $5.51(0.99)+/-0.118$ & $6.05(1.02)+/-0.256$ \\
Mean (SD, 95\% confidence interval) Comfort Score & $5.19(1.62)+/-0.193$ & $5.14(1.46)+/-0.366$ \\
\hline
\end{tabular}

The close similarity between these results for the Comfort subscale strongly suggests that attrition has not significantly affected the characteristics of the sample by (for example) introducing self-selection bias. However, this is less clear for the Knowledge subscale where there is at least a suspicion that the participants who already had the most personal knowledge of dementia were the ones most motivated to respond. This is intuitively credible and does not compromise the sample but leaves open the question of whether the participants who benefitted most from the process were those who already had awareness of some dimensions of dementia.

\subsection{Analysis}

Responses were scored on a 7-point Likert scale, with 'Strongly Agree' scoring 7 and 'Strongly Disagree' scoring 1. This gave a theoretical maximum of 56 for the Knowledge and 84 for the Comfort subscale, with a mid-point of 4 for each individual item. Negatively worded items were reverse coded before data were analysed. The scale scores for Knowledge and for Social Comfort were created by averaging scale items to produce a number between 1 and 7.

The appropriate methods of analysis for Likert-scale data have been the subject of some debate as they more closely resemble ordinal than interval data. The position taken here follows that of Carifio and Perla (Carifio and Perla 2008) in that the two subscales were treated as scale data suitable for parametric analysis whereas the individual items were treated nonparametrically. However, individual items were treated as yielding interval data for exploratory purposes, without attempting to draw statistical inferences from them (George et al. 2013).

\subsection{Hypothesis 1}

The subscales for Knowledge and Comfort demonstrated good internal reliability. Cronbach's alphas were: Comfort T1 .89, Knowledge T1 .79, Comfort T2 .88, Knowledge T2 .74, indicating acceptable reliability for both but more internal consistency for the Comfort subscales. Since examination of the Mean and Standard Deviation figures for each scale indicated the presence of significant negative skew, bootstrapping was performed to meet the assumptions of parametric testing.

Bootstrapped $(\mathrm{N}=1000)$ paired-samples $t$-tests were conducted on combined scores for the Knowledge and Comfort scales at T1 and T2 (Table 2). The results of these tests indicate 
that Hypothesis 1 should be retained in relation to the Knowledge and Comfort scales, but rejected for the individual Fear item.

Table 2. Results of analysis of scores at T1 and T2 for Knowledge and Comfort scales.

\begin{tabular}{|c|c|c|c|c|c|c|c|}
\hline \multirow[t]{2}{*}{ Time/Subscale } & \multirow[t]{2}{*}{ Mean } & \multirow[t]{2}{*}{ SD } & \multirow{2}{*}{$\begin{array}{l}\text { Difference } \\
\text { in Mean }\end{array}$} & \multirow[t]{2}{*}{ Cohen's d } & \multirow{2}{*}{$\begin{array}{c}\text { Sig. } \\
\text { (2-Tailed) }\end{array}$} & \multicolumn{2}{|c|}{$\begin{array}{c}\text { Bootstrap 95\% } \\
\text { Confidence Interval }\end{array}$} \\
\hline & & & & & & Lower & Upper \\
\hline T1 Comfort & 5.14 & 0.96 & \multirow{2}{*}{0.48} & \multirow{2}{*}{0.68} & \multirow{2}{*}{0.000} & \multirow{2}{*}{0.30} & \multirow{2}{*}{0.66} \\
\hline T2 Comfort & 5.61 & 0.85 & & & & & \\
\hline T1 Knowledge & 6.05 & 0.64 & \multirow{2}{*}{0.19} & \multirow{2}{*}{0.31} & \multirow{2}{*}{0.018} & \multirow{2}{*}{0.03} & \multirow{2}{*}{0.35} \\
\hline T2 Knowledge & 6.24 & 0.52 & & & & & \\
\hline
\end{tabular}

It is worthy of note that the effect size in the case of the Comfort scale $(9.3 \%$ mean increase) is substantial compared to that for the Knowledge scale (2.6\% increase). This may be evidence of a 'ceiling effect' for the Knowledge subscale, as the mean score before intervention was already above 6 out of a possible 7. A ceiling effect in turn may be due to the personalist nature of church congregational belonging when compared with day-to-day life; it is noteworthy that the scale's authors recently undertook an online study of the wider community in Wisconsin, and reported average Knowledge scores of only 5.89 (Ebert et al. 2019). However, both in their study and in George et al. (George et al. 2013), the Knowledge subscale scored more highly than the Comfort subscale, suggesting that it is intrinsically less sensitive. The difference may therefore be largely accounted for by artefacts of the scale.

\subsection{Hypothesis 2}

This hypothesis tested the theory that people who rated dementia as among their most-feared conditions would be less likely to be comfortable in the presence of people with dementia. We also hypothesised that there would be some correlation between fear of dementia and knowledge of its symptoms, although the direction of the relationship was harder to assess.

Nonparametric measures of correlation were used for a preliminary exploration of this hypothesis (Table 3). In the event, no consistent relationship was found between the Fear item and the two subscales, so we concluded that the item was of little or no value. A more sophisticated measure such as that used by Ebert et al. (Ebert et al. 2019) might with hindsight have yielded data of more significance.

Table 3. Spearman's rho correlation scores for Fear item against Knowledge and Comfort subscales.

\begin{tabular}{lccccc}
\hline & & T1 Comfort & T1 Knowledge & T2 Comfort & T2 Knowledge \\
\hline \multirow{2}{*}{ Fear T1 (N =61) } & Spearman's rho & -0.092 & -0.307 & & \\
& Sig. (2-tailed) & 0.479 & 0.016 & & \\
\hline \multirow{2}{*}{ Fear T2 (N=61) } & Spearman's rho & & & -0.209 & -0.178 \\
& Sig. (2-tailed) & & & 0.107 & 0.169 \\
\hline
\end{tabular}

\subsection{Exploratory Analysis of Individual Items}

Since it is not appropriate to analyse individual Likert items with parametric tools, an exploratory analysis was conducted using the Wilcoxon signed-rank test to identify those items that contributed most to the increase in subscale scores at T2 (Table 4). Where a significant change in score (z) was identified, effect size (Cohen's r) was calculated as $z / \sqrt{ } N$ where $N=61$ so $\sqrt{ } N=7.81$ 
Table 4. Exploratory analysis for each item at T1 and T2.

\begin{tabular}{|c|c|c|c|c|c|c|c|c|}
\hline Subscale & Question & $\begin{array}{c}\text { Median } \\
\text { (Interquartile) } \\
\text { at T1 }\end{array}$ & $\begin{array}{c}\text { Median } \\
\text { (Interquartile) } \\
\text { at T2 }\end{array}$ & $\begin{array}{c}\text { Mean } \\
\text { T1 }\end{array}$ & $\begin{array}{c}\text { Mean } \\
\text { T2 }\end{array}$ & $\begin{array}{l}\text { Wilcoxon } \\
\text { (z) }\end{array}$ & Probability & $\begin{array}{c}\text { Effect } \\
\text { Size (r) }\end{array}$ \\
\hline Comfort & 1 & $6(2)$ & $6(2)$ & 5.36 & 5.79 & 2.610 & 0.009 & 0.334 * \\
\hline Comfort & 2 & $6(2)$ & $6(1)$ & 5.41 & 5.93 & 2.666 & 0.008 & 0.341 * \\
\hline Comfort & 3 & $6(1)$ & $6(0)$ & 5.62 & 5.84 & 1.333 & 0.183 & \\
\hline Comfort & 4 & $5(2)$ & $6(1)$ & 4.97 & 5.57 & 3.587 & 0.000 & 0.459 * \\
\hline Comfort & 5 & $6(1)$ & $6(1)$ & 5.48 & 5.85 & 1.909 & 0.056 & \\
\hline Comfort & 6 & $6(3)$ & $6(2)$ & 5.44 & 5.82 & 2.202 & 0.028 & 0.282 \\
\hline Comfort & 7 & $7(1)$ & $7(1)$ & 6.61 & 6.54 & -0.816 & 0.414 & \\
\hline Comfort & 8 & $5(4)$ & 6(3) & 4.64 & 5.51 & 3.810 & 0.000 & $0.487^{*}$ \\
\hline Comfort & 9 & $5(3)$ & $5(2)$ & 4.75 & 5.13 & 2.103 & 0.035 & 0.269 \\
\hline Comfort & 13 & $5(2)$ & $6(1)$ & 5.05 & 5.67 & 3.781 & 0.000 & 0.484 * \\
\hline Comfort & 16 & $3(3)$ & $5(3)$ & 3.43 & 4.62 & 4.245 & 0.000 & $0.543^{* *}$ \\
\hline Comfort & 17 & $6(2)$ & $6(2)$ & 4.89 & 5.10 & 0.852 & 0.394 & \\
\hline Knowledge & 10 & $6(1)$ & $7(1)$ & 6.30 & 6.39 & 1.025 & 0.305 & \\
\hline Knowledge & 11 & $6(2)$ & $6(2)$ & 5.84 & 5.77 & -0.351 & 0.726 & \\
\hline Knowledge & 12 & $6(1)$ & $7(1)$ & 5.97 & 6.41 & 2.809 & 0.005 & 0.360 * \\
\hline Knowledge & 14 & $6(0)$ & $6(1)$ & 5.92 & 6.15 & 1.335 & 0.182 & \\
\hline Knowledge & 15 & $6(1)$ & $7(1)$ & 6.23 & 6.41 & 1.392 & 0.164 & \\
\hline Knowledge & 18 & $6(2)$ & $6(1)$ & 5.98 & 6.25 & 1.982 & 0.047 & 0.253 \\
\hline Knowledge & 19 & $6(1)$ & $6(1)$ & 6.23 & 6.36 & 1.171 & 0.242 & \\
\hline Knowledge & 20 & $6(1)$ & $6(1)$ & 5.92 & 6.18 & 1.744 & 0.081 & \\
\hline
\end{tabular}

${ }^{*}=$ medium effect size $(\mathrm{r}=0.3$ to 0.5$) .{ }^{* *}=$ large effect size $(>0.5)$.

\section{Discussion}

The key finding from the analysis of data in this paper is that the introduction of a Dementia-Friendly Church programme to Anglican congregations in the diocese under study was associated with a positive change in attitudes to people with dementia as measured by the Comfort subscale of the DAKS questionnaire. A smaller change was detected in the Knowledge subscale which, while still statistically significant, does not represent such a large effect size. As expected from comparison of the means, the items that contribute most to the change between T1 and T2 are grouped in the Comfort subscale. The mean change in score (0.48) represents a change per item of nearly half a point. The largest per-item change was for question 16, where the change was of more than one whole point on the scale. In descending order of importance, the five which manifested the largest change are:

16. I feel frustrated because I do not know how to help people with dementia.

8. I am not very familiar with dementia

13. I feel relaxed around people with dementia

4. I feel confident around people with dementia.

12. It is possible to enjoy the company of people with dementia.

These results suggest that, as a result of the programme, the 'average member of the congregation' is likely to feel more comfortable with members of the congregation or visitors who display symptoms of dementia. They are likely to feel less uncertain about how to respond, more relaxed and confident, and consequently more likely to enjoy associating with a person with dementia. However, there is much less evidence that the programme has led to a change in the level of knowledge in the congregations studied. As noted above, this may be a problem with the instrument used: as the level of background knowledge in British culture has risen, the tool may need revision to avoid ceiling effects in the data analysis. It may also reflect that fact that the primary purpose of DFCh has been to bring about a change in church culture and activity, with the acquisition of factual knowledge as a subsidiary means to that end. 
It is also noteworthy that the Fear item introduced on fear of dementia shed no useful light on how individual feelings were changing as a result of the programme. There was certainly change in individuals' answers, as respondent-by-respondent examination shows, but these did not add up to a change in the overall scores. This might of course be because the item shows poor test-retest reliability, so that random fluctuations in the answers at the two time points overwhelm any substantive changes in the data. It is noteworthy that when Ebert et al. used a more sophisticated and sensitive measure of fear using 11 statements regarding concerns for developing dementia selected from the Fear of Alzheimer's Disease Scale (French et al. 2012), they found that lower levels of dementia fear significantly predicted for higher levels of comfort, a finding not replicated in this smaller and more focused study (Ebert et al. 2019). Another possibility is that the changes are cancelling each other out: for some people, the programme may reduce their fear at the prospect of a dementia diagnosis, while for others, the increasing awareness increases it. The data do not allow further investigation of this possibility, but some other studies provide some supporting evidence (Cantegreil-Kallen and Pin 2012). There is no way to decide this issue based on the present dataset, but further studies may illuminate the relationship between Fear, Knowledge and Comfort with more clarity.

\section{Conclusions}

The main finding of this study is that the DFCh intervention in the test diocese leads to a measurable improvement in the level of comfort experienced by participants in the presence of people living with dementia. Some caution is appropriate here, because the absence of a control group and the high rate of attrition between first and second measurements both limit the degree to which it has been possible to control for confounding variables, but the effect size is noteworthy for a brief intervention of this type. It is, however, as yet unclear how DFCh brings about this change of attitudes. For example, what proportion of the change is simply a result of increased attention being given to dementia at congregational level, with dedicated events and named representatives in the church, and what proportion depends upon specific content that can be added to or improved to bring about more change? Will further activity bring about more beneficial change, and if so, in what way?

It is also unclear at present how this beneficial change to individual participants translates into a cultural change across the church community as a whole. It is very likely that this change in the attitudes of participating individuals will be expressed in a more open, welcoming and understanding culture towards people living with dementia and their companions, but it is hard to measure how a collective 'culture' changes as the result of an intervention such as the DFCh programme, so further insight into the nature of this culture change will need to await the findings of a subsequent, qualitative study.

Nevertheless, it can be concluded with confidence that a structured programme of this type is associated with a significant shift in attitudes among members of religious congregations. As intentional communities, such congregations provide a 'captive audience' who expresses a shared identity, values and a degree of commitment to each other; most also express some commitment to the wellbeing of the wider community in which they are embedded. These features no doubt predispose the congregation towards a favourable outcome and prepare it to spread a beneficial attitudinal change more widely (Blanchard et al. 2008). There is an abundance of evidence that religious communities can be deployed to spread health promotion messages and encourage beneficial behaviour changes (Taylor et al. 2007; Blanchard et al. 2008; Local Government Association 2017; Brewer 2019; Long et al. 2019); in a similar way, we conclude that cultural attitudes towards people with dementia may be beneficially influenced by targeting key messages at faith-based communities.

We may also conclude, therefore, that structured interventions to change attitudes could have wider application in supporting other types of 'intentional' communities in their efforts to become more open and accommodating to people with dementia. This certainly includes faith-based organisations such as Dementia Friendly Gurudwaras (Dementia Friendly Gurudwaras 2014), but might also apply to support groups (such as the British Legion and Alcoholics Anonymous), sporting organisations 
(such as golf clubs) and community networks (such as the Polish Association). A pattern of focused intervention, repeated at a few events, among people who meet together anyway and are used to discussing things together, may prove a useful tool in developing more dementia-friendly communities.

Author Contributions: Conceptualization, D.P. and P.K.; methodology, P.K.; validation, D.P. and P.K.; formal analysis, P.K.; investigation, D.P. and P.K.; data curation, P.K.; writing-original draft preparation P.K.; writing-review and editing, D.P. All authors have read and agreed to the published version of the manuscript.

Funding: This research received no external funding

Acknowledgments: Thanks are due to the three Dementia-Friendly Church Enablers of the Diocese of Lichfield, and particularly to Sarah Thorpe, who took lead responsibility for the majority of the parish-level data gathering. She is also the main author of much of the content of the Dementia Friendly Churches programme. Thanks also to Emeritus Professor Susan McFadden, for permission to use the DAS in this study.

Conflicts of Interest: The authors declare no conflict of interest.

\section{References}

Alzheimer's Society. 2019. What is a Dementia Friendly Community? Available online: https://www.alzheimers. org.uk/get-involved/dementia-friendly-communities/what-dementia-friendly-community (accessed on 24 February 2020).

Alzheimer's Society. 2020. List of Dementia Friendly Communities. Available online: https://www.dementiafriends. org.uk/WebArticle?page=dfc-public-listing\#.XIPA-sN2uta (accessed on 24 February 2020).

Alzheimer Disease International. 2016. Dementia Friendly Communities Key Principles, Alzheimer's Disease International, London. Available online: https://www.alz.co.uk/adi/pdf/dfc-principles.pdf (accessed on 27 May 2020).

Blanchard, Troy C., John P. Bartkowski, Todd L. Matthews, and Kent R. Kerley. 2008. Faith, Morality and Mortality: The Ecological Impact of Religion on Population Health. Social Forces 86: 1591-620. [CrossRef]

Blendon, Robert J., John M. Benson, Elizabeth M. Wikler, Kathleen J. Weldon, Jean Georges, Matthew Baumgart, and Beth A. Kallmyer. 2012. The impact of experience with a family member with Alzheimer's disease on views about the disease across five countries. International Journal of Alzheimer's Disease. [CrossRef]

Brewer, LaPrincess C. 2019. We've ComeThis Far by Faith: The Role of the Black Church in Public Health. American Journal of Public Health 109: 385-86. [CrossRef]

Buckner, Stefanie, Nicole Darlington, Michael Woodward, Marina Buswell, Elspeth Mathie, Antony Arthur, Louise Lafortune, Anne Killett, Andrea Mayrhofer, John Thurman, and et al. 2019. Dementia friendly communities in England: A scoping study. International Journal of Geriatric Psychiatry 34: 1235-43. [CrossRef] [PubMed]

Cantegreil-Kallen, Inge, and Stéphanie Pin. 2012. Fear of Alzheimer's disease in the French population: Impact of age and proximity to the disease. International Psychogeriatrics 24: 108-16. [CrossRef] [PubMed]

Carifio, James, and Rocco Perla. 2008. Resolving the 50-year debate around using and misusing Likert scales. Medical Education 42: 1150-52. [CrossRef]

Dean, Janet, Kay Silversides, Janet Crampton, and Julie Wrigley. 2015a. Evaluation of the Bradford Dementia Friendly Communities Programme Programme. JRF Findings. Available online: https://www.jrf.org.uk/ report/evaluation-bradford-dementia-friendly-communities-programme (accessed on 27 May 2020).

Dean, Janet, Kay Silversides, Janet Crampton, and Julie Wrigley. 2015b. Evaluation of the York Dementia Friendly Communities Programme Programme. JRF Findings. Available online: https://www.jrf.org.uk/report/ evaluation-york-dementia-friendly-communities-programme (accessed on 27 May 2020).

Dementia Friendly Gurudwaras. 2014. A Toolkit to Making Your Local Gurudwara Dementia Friendly. London: Dementia Friendly Gurudwaras, Available online: https://dementiafriendlygurudwaras.com/ (accessed on 27 May 2020).

Department of Health. 2015. Prime Minister's Challenge on Dementia 2020; London: Department of Health. Available online: https://www.gov.uk/government/publications/prime-ministers-challenge-on-dementia (accessed on 27 May 2020).

Ebert, Alexandria R., Danica Kulibert, and Susan H. McFadden. 2019. Effects of dementia knowledge and dementia fear on comfort with people having dementia: Implications for dementia-friendly communities. Dementia. [CrossRef] 
Epps, Fayron, Glenna Brewster, Karah Alexander, Jenny Choe, Virginia Heidbreder, and Kenneth Hepburn. 2019. Dementia-friendly faith village worship services to support African American families: Research protocol. Research in Nursing and Health 42: 189-97. [CrossRef]

Epps, Fayron, Virginia Heidbreder, Karah Alexander, Audrey Tomlinson, Valencia Freeman, and Nancy Williams. 2020. A dementia-friendly church: How can the African American church support families affected by dementia? Dementia. [CrossRef]

Farr, Emma-Dawn, and Jennifer Bute. 2015. Dementia: Good living in our churches. Working with Older People: Community Care Policy E Practice 19: 134-39. [CrossRef]

French, Samantha L., Mark Floyd, Stacy Wilkins, and Sheryl Osato. 2012. The Fear of Alzheimer's Disease Scale: A new measure designed to assess anticipatory dementia in older adults. International Journal of Geriatric Psychiatry 27: 521-28. [CrossRef]

Garland, Rodie. 2017. Building Dementia-Friendly Faith Communitiies. London: Faith Action Network, Available online: https://www.faithaction.net/portal/wp-content/uploads/fa-dementia-friendly-faith-communities.pdf (accessed on 27 May 2020).

George, Daniel R., Heather L. Stuckey, and Megan M. Whitehead. 2013. An arts-based intervention at a nursing home to improve medical students' attitudes toward persons with dementia. Academic Medicine 88: 837-42. [CrossRef] [PubMed]

Goldenberg, Naomi R. 2015. The Category of Religion in the Technology of Governance: An Argument for Understanding Religions as Vestigial States. In Religion as a Category of Governance and Sovereignty. Edited by Stack Trevor, Naomi Goldenberg and Timothy Fitgerald. Leiden: Brill, pp. 280-92.

Hattink, Bart, Franka Meiland, Henriëtte van der Roest, Peter Kevern, Francesca Abiuso, Johan Bengtsson, Angele Giuliano, Annalise Duca, Jennifer Sanders, Fern Basnett, and et al. 2015. Web-based STAR E-learning course increases empathy and understanding in dementia caregivers: Results from a randomized controlled trial in the netherlands and the United Kingdom. Journal of Medical Internet Research 17. [CrossRef] [PubMed]

Hebert, Catherine A., and Kezia Scales. 2019. Dementia friendly initiatives: A state of the science review. Dementia 18: 1858-95. [CrossRef] [PubMed]

Heward, Michelle, Anthea Innes, Clare Cutler, and Sarah Hambidge. 2017. Dementia-friendly communities: Challenges and strategies for achieving stakeholder involvement. Health and Social Care in the Community 25. [CrossRef]

Institute of Public Care. 2015. Evaluation of Hampshire Dementia Friendly Communities: Final Report. Available online: http://ipc.brookes.ac.uk/publications/pdf/Hampshire_Dementia_Friendly_Communities_ Evaluation_June_2015.pdf (accessed on 27 May 2020).

Jewell, Albert, John Cole, Jenny Rolph, and Paul Rolph. 2016. The faith of primary carers of persons with dementia. Journal of Religion, Spirituality and Aging 28: 313-37. [CrossRef]

Jewell, Albert, John Cole, Paul Rolph, and Jenny Rolph. 2017. The Loneliness of the Long-distance Carer: The Experience of Primary Carers of Loved-Ones with Dementia. Rural Theology 15: 97-112. [CrossRef]

Kevern, Peter. 2009. The grace of foolishness: What Christians with dementia can bring to the churches. Practical Theology 2: 205-18. [CrossRef]

Lin, Shih-Yin. 2017. "Dementia-friendly communities" and being dementia friendly in healthcare settings. Current Opinion in Psychiatry 30: 145-50. [CrossRef]

Local Government Association. 2017. Working with Faith Groups to Promote Health and Wellbeing; London: Local Government Association. Available online: https://www.local.gov.uk/working-faith-groups-promote-healthand-wellbeing (accessed on 27 May 2020).

Long, Katelyn NG, Ryan J. Gregg, Tyler J. VanderWeele, Doug Oman, and Lance D. Laird. 2019. Boundary crossing: Meaningfully engaging religious traditions and religious institutions in public health. Religions 7: 412. [CrossRef]

Márquez-González, María, Javier López, Rosa Romero-Moreno, and Andrés Losada. 2012. Anger, Spiritual Meaning and Support from the Religious Community in Dementia Caregiving. Journal of Religion and Health 51: 179-86. [CrossRef]

O'Connor, Melissa L., and Susan H. McFadden. 2010. Development and psychometric validation of the dementia attitudes scale. International Journal of Alzheimer's Disease. [CrossRef]

Plunkett, Robyn, and Peter Chen. 2016. Supporting healthy dementia culture: An exploratory study of the church. Journal of Religion and Health 55: 1917-28. [CrossRef] [PubMed] 
Post, Stephen G. 2000. The concept of Alzheimer disease in a hypercognitive society. In Concepts of Alzheimer Disease: Biological, Clinical and Cultural Perspectives. Baltimore: Johns Hopkins University Press, pp. $245-56$. Swinton, John. 2017. Becoming Friends of Time: Disability, Timefullness, and Gentle Discipleship. London: SCM Press. Taylor, Robert Joseph, Linda M. Chatters, and James S. Jackson. 2007. Religious and Spiritual Involvement Among Older African Americans, Caribbean Blacks, and Non-Hispanic Whites: Findings from the National Survey of American Life. The Journals of Gerontology 62: S238-50. [CrossRef] [PubMed]

(C) 2020 by the authors. Licensee MDPI, Basel, Switzerland. This article is an open access article distributed under the terms and conditions of the Creative Commons Attribution (CC BY) license (http://creativecommons.org/licenses/by/4.0/). 